\title{
SOBRE ANGELOLOGÍA Y PROFECÍA EN MAIMÓNIDES
}

\author{
Antonio Ríos Rojas \\ Universidad de Salamanca
}

\section{RESUMEN}

La figura del ángel, en las múltiples formas en que éste aparece tanto en la Escritura como en la filosofía, es justamente una realidad trazadora de límites en el entendimiento humano, al tiempo que traspasa naturalmente tal entendimiento, llevando a éste a su culmen en la intuición y en el conocimiento profético. La visión de la realidad angélica por parte de Maimónides alcanza estos mismos logros en la gnoseología, pero además alcanza cotas nuevas - ya vislumbradas en el mundo presocrático-, respecto a la metafísica y al sentido de lo vivo.

Palabras clave: Maimónides, ángel, profecía.

\begin{abstract}
The image of the angel in the various forms which appears both in the Holy Bible and in Philosophiy is exactly a reality that draws the limits of human understanding. At the same time, it naturally transfixes such understanding, carrying the latter to its climax in intuition and prophetic knowledge. Maimonides' vision of angelic reality reaches these very same achievemants in gnoseology but furthermore it reaches new dimensions. The latter had already been glimpsed at in the presocratic world in relation to metaphysics and the sense of the living.
\end{abstract}

Key words: Maimonides, angel, prophecy.

\section{INTRODUCCIÓN}

Una visión profunda de la angelología nos lleva a tratar a ésta como uno de los temas que adquieren mayor importancia dentro de la filosofía medieval; esta importancia radica en servir como posible solución a un problema gnoseológico fundamental y que en expresión de Gilson podríamos expresar así: ¿Es Dios objeto natural de nuestro intelecto?. Las bifurcaciones a esta pregunta fundamental están también trazadas, pues gracias a ella nos corresponde preguntarnos si realidades tales como el Bien, la Verdad y la Unidad son igualmente objeto natural de nuestro intelecto. De buenas a primeras, ya tenemos delante de nosotros a los mismos trascendentales, que a modo distinto del pensamiento griego, bañan todo el pensamiento medieval. 
Hemos entrado en un modo de enfocar lo que he llamado el problema gnoseológico fundamental. Hay otro modo de expresar este problema y puede ser este: ¿De qué modo es pensable que dos seres, uno infinito y absoluto como es Dios, y otro, finito y relativo como es el hombre, entren en contacto?.' En multitud de ocasiones se ha argumentado que el punto de unión entre el hombre y Dios es precisamente la vida intelectiva; ${ }^{2}$ podría ser ése, pues, el punto de unión; pero también se nos ha puesto de manifiesto en multitud de ocasiones que el entendimiento del hombre difiere esencialmente del de Dios hasta el punto de ser algo separado y activamente diverso. Dios conoce los individuos conociendo las especies, digamos que el todo es anterior a la partes en el conocimiento de Dios; así es su modo de conocer. El entendimiento del hombre, en cambio, es discursivo, necesita de las partes.

En base a esto se ha dicho que Dios no tiene contacto con la materia, pues esto rebajaría su esencia. Pero tampoco tendría contacto con el intelecto humano precisamente por lo mismo.

$\mathrm{Ni}$ que decir tiene que estos no son los motivos de que hayan ángeles en la Escritura, esos motivos harían que nos introduzcamos, entre otros temas, en la fenomenología religiosa y bíblica de forma específica eso nos llevaría a campos de estudio que en nada son míos, pero sí afirmaremos -entrando ahora en lo que ya es nuestro tema - que dada la existencia de los ángeles en la Escritura, éstos van a ser aprovechados para explicaciones importantes en la teoría del conocimiento. Así pues, la figura del ángel va a significar el contacto, la vía posible entre Dios y el hombre; además, en el pensamiento judío esto se acrecienta aún más, ya que no existe el concepto de encarnación - al menos en su realización-, concepto clave que sí posee el cristianismo. Este contacto o vía entre el hombre y Dios supone ni más ni menos que una limitación en el conocer humano.

El vislumbramiento de los límites de nuestro intelecto se encuentra ya en Aristóteles, el cual formuló una separación en el intelecto mismo: el agente y el paciente, el primero siempre en acto, el segundo en potencia de ser actualizo. ¿Cómo acontece la relación de uno con respecto al otro?: por participación dirá la interpretación neoplatónica; de ahí que esa participación fuese entendida como mediación, ya que en el sistema de emanaciones neoplatónico, el intelecto agente era de las primeras realidades en emanar. ${ }^{3}$ De ahí que la función del intelecto agente fuese angélica -ya veremos la función del ángel como mediación o mediador - y autores como Gabirol, Maimónides, Ezra o Al-Farabi entendieran el intelecto agente como realidad angélica - y no sólo en modo comparativo, como tendremos ocasión de probar en Maimónides-.

1 Nótese que estamos en un plano donde vamos a suponer que esa realidad que llamamos Dios; y que difiere de la realidad humana, existe. Lo damos por supuesto como punto de partida del presente estudio. Es una suposición que ya se encuentra en el primer modo de expresar esa pregunta gnoseológica fundamental, sino ¿de qué otro modo sino del natural accedemos a la comprensión de las cosas?. Tal suposición se hace más explícita en el segundo modo de expresar el problema gnoseológico. Quisiera también expresar que tal suposición no es gratuita, sino que nos pone en mayor situación de conocimiento de este mundo medieval, donde la existencia de Dios vista desde lo que Severino y Zubiri han llamado el horizonte de la nihilidad, se encuentra en mayor o menor grado supuesta. Sea la operación gnoseológica "de arriba abajo" o de "abajo arriba", el horizonte de la nihilidad que es el horizonte del creacionismo realiza esta suposición indicada.

2 Cf. Maimónides, Guía de perplejos. Madrid, Trotta. cap 1, I parte, donde se afirma que es por la razón por lo que el hombre es imagen y semejanza de Dios.

3 Pasamos por encima la particularidad de los diversos sistemas; hagamos notar que por ejemplo para Ibn Gabirol es la materia y la forma lo primero creado, produciéndose de ahí la continuidad en la línea emanatista; que en Al-Farabi, son las inteligencias de las esferas y posteriormente el intelecto agente. 
Este problema gnoseológico fundamental es expresado de forma muy concisa e interesante en un estudio de Santiago Fernández Burillo, donde se hace alusión a que entender y razonar responden al movimiento de construir unidades. ${ }^{4}$ Pues bien, desde mi punto de vista, lo máximo a que se llegó en esta cuestión( si no fue a lo máximo, fue a lo máximo que se dejó llegar) de construir unidades, tanto generalmente en el pensamiento griego como en el medievo, fue al concepto de substancia primera; en cambio, la unidad absoluta, pura, en el conocer se dejó sólo a Dios. Ahora bien, que no pueda el hombre acceder a la unidad isignifica que esta no exista?. Esta pregunta que viene siendo formulada desde el pensamiento presocrático es solventada así en la alta Edad Media : el hombre puede acceder a la unidad, como a la verdad y al bien, pero no por su razón discursiva, sino por la intuitiva. De esta forma ejerció el neoplatonismo una decisiva influencia en autores judíos y árabes, los cuales vieron que la única forma de posibilidad del hombre respecto a lo intuitivo venía dado de fuera, en este caso era el ángel el portador de la sabiduría, y por ende de los trascendentales, y de cada misterio que osase postrarse ante el hombre medieval.

Desde esta introducción de corte gnoseológica se desarrollarán los tres apartados siguientes, el primero de los cuales dedicaré a enlazar la angelología con uno de los temas desde los que adquiere sentido en la Guía de perplejos, y no es otro que el tema de la profecía, el segundo apartado trata de solucionar los problemas gnoseológicos apuntados ya con anterioridad tanto en la introducción como en el apartado primero, todo esto en confrontación directa con la Guía de perplejos; el tercer y último apartado es un ensayo de connotaciones metafísicas desde el que pretendo hacer ver una línea común desde un aspecto de la angelología en Maimónides con la filosofía de Baruc de Spinoza.

Antes de nada, y como conclusión a esta introducción, nos acercaremos de forma breve a la figura de los ángeles en el Antiguo Testamento.

Si nos centramos en la Escritura, el ángel es un mensajero de Dios, un intermediario entre Dios y el hombre; la figura del profeta es igualmente la de mediador, pero éste no sería tal si a su vez no tuviese la mediación que es el ángel, el cual toma en la Escritura diversas formas: fuerza, fuego, seres con rostros diversos. En el Antiguo Testamento la naturaleza angélica no se describe puntualmente, tan sólo quedan descritas las acciones de estas realidades angélicas, en este sentido, una descripción del ángel siempre existe, descripción que en la mayoría de ocasiones se hace desde posturas antropomórficas ${ }^{5}$ incluso con formas naturales como el fuego, o con miembros del género animal como es el caso de las alas e incluso de algunos rostros animales como acontece en las visiones de Ezequiel, ejemplo que de forma concisa trae Maimónides y que también nosotros comentaremos. En ocasiones se habla también del ángel con un concepto tan equívoco -que también intentaremos dilucidar - como el de "fuerza"; e incluso se emplea en múltiples

4 Fernández Burillo, S: "Inteligencias separadas y racionalismo medieval" en Actas del I Congreso Nacional de Filosofia Medieval. Zaragoza,1992, pp. 261-271.

5 En más de una ocasión nos advierte Maimónides que la Torah está escrita en lenguaje humano e igualmente al modo cognoscitivo de los hombres, Cf. Maimónides: Guía de perplejos. Madrid, Trotta, cap.59, I parte, p.161, no obstante, esto no aduce a no profundizar en los sentidos alegóricos de la mayor parte de los pasajes, una defensa radical de la alegoría se encuentra ya en el mismo prólogo esp, pp. 55-60. 
ocasiones en la Escritura la palabra Elohim - que suele ser apelativo de Dios- para denominar al ángel. ${ }^{6}$

Pero insistimos en que es su acción la que se nos quiere dar a conocer, no su naturaleza; en este sentido tampoco Dios puede ser conocido por sus atributos ya que estos no indican una esencia, sino que sus atributos son sólo de acción, nunca atributos esenciales.?

La función del ángel es ser intermediario entre Dios y el hombre; de hecho el original hebreo "malak" significa mensajero con lo que el mismo término queda expresado a nivel de funcionalidad. La mediación del profeta no es tan directa como lo es la del ángel, este ángel se presenta a quien Dios quiere y de forma imprevisible portando un mensaje o siendo un ejecutor de la voluntad de Dios. ${ }^{8}$ El ángel puede transmitir mensajes muy distintos: de consuelo, mensajes en orden a interpretar la historia, mensajes que ordenan actuación a los hombres(especialmente de los profetas)....; pese a que hemos advertido que toman figura humana en sus apariciones, se apunta que su naturaleza es espiritual, especialmente en el desarrollo de la angelología talmúdica (S.V d.C) se hace hincapié en este aspecto. Esta naturaleza espiritual se pretende manifestar no asignándoles espaldas, de esta forma se indica que constantemente ven a Dios ${ }^{10}$ en el sentido de que son por él y están para realizar siempre mandatos, órdenes divinas ${ }^{11}$ Dios es su Señor, así interpreta Maimónides el aserto que dice "Dios es el Señor de los dioses"; puesto que los hombres son de un rango muy inferior para ser parangonados con Dios ${ }^{12}$ sobre este mismo tema algunos exégetas han interpretado aquella frase de Gn 1 "Hagamos al hombre a nuestra imagen y semejanza" como si ciertos testigos (los ángeles) contemplaran y aprobaran la obra creadora de Dios, ya que fueron creados antes que los hombres. ${ }^{13}$

\section{LA ANGELOLOGÍA DESDE LA PROFECÍA}

La advertencia que de forma específica, nos da Maimónides en la Guía sobre las realidades angélicas, es aquella, según la cual, la visión de los ángeles sólo acontece en visión profética, ésta

6 Y es que la función mediadora del ángel es a veces tan directa y fielmente portadora de la voluntad de Dios, que, en efecto, en ocasiones se funden la realidad de Dios (Elohim) con la del ángel; Maimónides nos trae un ejemplo de este particular (Cf. O.c. Cap.27, I parte) refiriéndose al capítulo 31 del Génesis, allí se expresa jacob de esta forma: "... el el ángel del Señor me dijo en el sueño: Jacob... y yo le respondí: Heme aquí". Pero a todo esto al final de la interlocución se expresa ese ángel de esta forma: "yo soy el Dios que se te apareció en Betel y a quien ofreciste tus votos". Queda claro que es a Dios y no al ángel a quien Jacob ofrece sus votos, pero Jacob accede a tales revelaciones en visión profética y desde este punto de vista también aparece en la Escritura que el ángel es Dios (Elohim), esto es, directa y fielmente el portador de su palabra, desde este punto de vista defiende Maimónides a Onquelos cuando identifica Elohim con el ángel porque respeta profundamente la visión profética en la que se mueve Jacob.

7 Un desarrollo de lo que venimos comentando se encuentra en Maimónides: o.c.cap 54 y 58 de la I parte.

8 Cf. Maimónides: o.c, p.256: “...pues todos ellos ejecutan un mensaje soberano"

9 Para una más detallada lista de las funciones de los ángeles, cf. Sicre, J.L. Profetismo en Israel, ed. Verbo divino; pg.69-70.

10 Cf. Gonzalo Rubio C: La angelología en la literatura rabínica y sefardí; Barcelona, Riopiedras; p.40.

11 Apunta la autora antes citada que sólo en ocasiones estos ángeles desobedecen a Dios produciéndose el castigo de este a aquellos p.e. en $\mathrm{Gn} 50,68$ donde se dice que expulsó del cielo durante ciento treinta y ocho días a dos ángeles porque descubrieron prematuramente el decreto de la destrucción de Sodoma.

12 Cf. Maimónides: o.c. p.253.

13 Cf. Gonzalo Rubio:0.c p.38. 
puede acontecer tanto en sueños como en estado de vigilia. "Toda visión del mismo — del ángeltiene lugar en visión profética y conforme al estado de quien la aprende". ${ }^{14}$ No es este el lugar específico para tratar la profecía en Maimónides, pero echemos un vistazo a su definición: "emanación de Dios mediante el intelecto activo sobre la facultad racional en primer término y seguidamente sobre la imaginativa". ${ }^{15}$

Desde este punto de vista tienen una esencial importancia tanto la racional como la imaginativa; para que ambas puedan llegar a su cumbre, necesitan de una preparación mediante la actuación recta (ética) conforme a la Escritura (torah)y también mediante el estudio encaminado a la metafísica (al cual le es necesario pasar por la lógica, las matemáticas, etc.); esto es, la profecía no adviene como exclusivo don de Dios, sino que en primer lugar el receptáculo debe estar preparado ${ }^{16} \mathrm{La}$ profecía, pues, es visión; ésta lo es de acontecimientos futuros, o como es nuestro caso, de realidades espirituales; esta visión gracias a la facultad imaginativa, trabaja al modo de la visión humana, y ve esas realidades "como si tuvieran una real correspondencia en el mundo exterior", esa correspondencia es ganada mediante la extensión, mediante la forma; de este modo en la visión profética, el profeta ve a las realidades angélicas como si poseyeran formas tanto humanas como animales, como de cualquier otro ser corpóreo, por ejemplo el fuego. Así pues, es inherente a la visión de las realidades angélicas operar conforme al conocimiento propio del hombre, necesitado pues de extensión incluso para conocer las realidades espirituales, ${ }^{17}$ este no deja de ser un signo más de la profunda diferencia entre los seres espirituales y el hombre. Es fruto precisamente de esta finitud del hombre el dejarse llevar por un mal uso de la imaginativa, ya que ésta puede originar mil combinaciones entre cosas ya presentes, ya ausentes; es por ello por lo que nuestro autor insiste fuertemente en un buen uso de la imaginativa, buen uso que se gesta en discernir bien entre lo que pertenece a lo inteligible y a lo meramente imaginario, ocasionador de quimeras y fantasías. ${ }^{18}$

Una vez que estamos advertidos de estos particulares, también habremos de tener en cuenta el estado intermedio de los ángeles respecto de Dios y de los hombres. Los ángeles son réalidades espirituales, exentas de materia, son además creados por Dios; con esto imprime Maimónides en las realidades angélicas un más allá dentro del mero emanatismo que en infinitas ocasiones se deja translucir en la obra; de forma concisa nos dice el autor que son mensajeros de Dios al hombre, se crean pues los ángeles con el fin de ser intermediarios, de ser una vía entre el hombre y Dios. Ahora bien, ese carácter de ser mensajero no quiere decir que Dios dé órdenes directamente a los ángeles

14 Maimónides: o.c. cap 6, II parte, p.256.

15 Cf. O.c. : cap.36, II parte, p.334-335.

16 Más adelante discutiremos este punto de si la profecía adviene o no por la voluntad de Dios cuando indaguemos más sobre las realidades angélicas.

17 Esto es importante en la Guía ya que incluso a Dios sólo se le puede conocer por sus acciones, no por su esencia, ya que la faz de Dios no la verá nadie, sólo se accede a su nombre Yahve para indicar su esencia, pero sólo para indicarla, conocerla se torna imposible al modo de conocer discursivo del hombre. Incluso cuando se habla de teología negativa no es más que una salvedad para no asignar atributos divinos, pero la teología negativa no es tampoco conocimiento, es tan sólo una forma no errónea de hablar; el verdadero conocimiento de Dios es de sus acciones con respecto al mundo y especialmente en la creación, con lo que se asigna una especie de bondad en la misma que viene a hacer una función más explícita — pero en el fondo la misma - que el trascendental "Bonum".

18 Cf. Especialmente este peligro en el uso de la imaginativa, sin discernimiento de lo que es posible e imposible en la lucha de Maimónides con los mutacálimes assaries; Maimónides: o.c. p.214-218. 
para que les sean comunicadas a los hombres, así nos lo hace ver en las diez primeras líneas de la página 137, y aunque posteriormente mantenga nuestro autor el término "mensajero", ${ }^{19}$ éste adviene desde una interpretación realmente portentosa que veremos posteriormente.

En las visiones de los ángeles acontece que en ocasiones tienen figuras de animales irracionales como por ejemplo las alas, ya lo indicamos anteriormente; esto indica en primer lugar que son inferiores a Dios y en segundo lugar que tienen la facultad del vuelo que más que facultad en el ángel es indicada como idea que espiritualiza al ángel y lo aleja de la realidad mundana. ${ }^{20}$ Pero el problema se agranda cuando Maimónides ensancha su concepto de ángel; porque esta realidad separada, exenta de materia, puramente espiritual se ha aplicado al ángel en tanto que inteligencia separada, pero también el término posee en la escritura otros asertos y son —además de las inteligencias separadas: al profeta, a cualquier tipo de mensajero e incluso a todo tipo de facultades, que Maimónides llama "animales", pero que luego amplía a todo tipo de facultad e incluso a todo tipo de "fuerza natural".

A continuación me dispongo a traer una de las interpretaciones de la visión en profecía de una realidad angélica, para así hacernos una idea no sólo del método alegórico en Maimónides, sino también para insertarnos en la visión escriturística con respecto a las realidades angélicas.

El relato bíblico en el que más se extiende Maimónides para explicar la visión de ángeles por parte del profeta es el perteneciente a Ez,1. No es ni mucho menos mi intención realizar un estudio exegético del pasaje, sino tan sólo traer una muestra del acceso del hombre a las realidades angélicas, así como de la acción de estas realidades.

A Ezequiel le aparecen cuatro figuras, cada una de ellas con cuatro rostros: de hombre por delante de la cabeza, de buey por la izquierda, de águila por detrás y de león por la derecha. El movimiento de cada uno de estos seres era recto e impulsado por el espíritu de Dios: “...iban a donde el espíritu los dirigía, sin volverse de espaldas". Fijémonos cómo se reduce incluso la volición de estas realidades, una vez que el espíritu los dirige no pueden volverse de espaldas. ${ }^{21} \mathrm{La}$ cábala pese a su misticismo de fondo, supone desde cierta comprensión, un intento de hacer del hombre el centro desvelador y cognoscitivo del mundo (de todos es conocido su intento de permutar la realidad - 0 en otros casos simplemente de desvelarla— que en sí esconde "el secreto" en la grafía y en los números); claro está que desde esta comprensión, los ángeles se convierten en meros instrumentos de los hombres para hacer la voluntad de éstos, no la de Dios. Si toda esta literatura cabalística hubiera comenzado en el S. XVI hablaríamos de un intento de desteocentrar a Dios, pero es en el S. VIII donde aparece, ¿quizás este intento de hacer del hombre el centro del mundo sólo acontece verdaderamente en la E. Media en la cábala y en la magia? Podría ser. Este volverse, ha sido, en cambio, en todo tipo de literatura exponente de la libertad humana, que no puede acatar una orden de lo alto sin dudar de ella, los ejemplos más claros de esto son Lot en el judaísmo y Orfeo en el

19 Cf. Cap.6, II parte; p.254.

20 Cf. Cap. 49, I parte, p.137.

21 Esta visión bíblica y también talmúdica desde la cual se entienden las realidades angélicas como servidoras de Dios, es de algún modo transferida en cierta comprensión por parte de la cábala, donde los ángeles están más bien al servicio de la invocación del hombre. (Cf. Gonzalo Rubio, C: o.c. p.57.). La autora citada nos comenta cómo en el Sefer Raziel -obra cabalística ya del S.XIII- los ángeles son invocados incluso para alcanzar la profecía. 
mundo griego. Por lo tanto lo primero que tenemos es la dependencia de los ángeles con respecto a Dios, la obediencia absoluta de la voluntad divina. Si solamente tuviéramos esta visión del ángel en Maimónides asentiríamos en que no aporta nada nuevo a estas realidades; en efecto, en esta visión de Ezequiel el ángel es tratado como una inteligencia separada, espiritual, creada para ser intermediario directo entre el hombre y Dios; pero como hemos apuntado ya, citando a Maimónides, el concepto de ángel se nos va a ir ampliando; en nuestro próximo apartado, indagaremos más en todo lo que nos aporta Maimónides a la angelología, sus ganancias con respecto a su misma obra e incluso nos atreveremos a sacar posibles consecuencias en la metafísica moderna.

\section{FUNCIONES DE LA REALIDAD ANGÉLICA. PLANTEAMIENTO GNOSEOLÓGICO}

No es aquí el lugar adecuado para traer toda la problemática entre fe y razón en la Edad Media, pero baste apuntar el intento de coordinar estos dos modos de conocimiento que al final llegan a la misma conclusión como son la razón y la revelación, intento que se pone muy especialmente de manifiesto en el final de la I parte de la Guía y en el comienzo de la II parte. El intento por hacer coincidir el aristotelismo (de corte neoplatónico) con la revelación, se manifiesta muy claramente en la angelología. Nótese que de hecho el tratamiento de las realidades angélicas en la Guía de perplejos sigue y es prácticamente consecuencia del tratamiento del tema de la astronomía, de las esferas y de sus inteligencias, tema que, si bien se encuentra en Aristóteles en el tratado de Coelo y en el cap XII de la Metafísica, es tratado por Maimónides claramente desde la interpretación aristotélica de Al Farabi: neoplatonismo.

El primer punto de unión entre estos dos accesos a la verdad es aquel que compara las inteligencias separadas con los ángeles; si las inteligencias son motor de las esferas, de la materia, son pues el principio del movimiento de una materia concreta, los ángeles tienen esa misma función y así los define concretamente: "fuerza ejecutora del movimiento"; el motivo por el cual lós ángeles son "ejecutores", "mensajeros" es el que ya apuntamos al comienzo de este escrito: Dios no puede ejecutar actos que le hagan entrar en contacto directo con la realidad material, de tal modo que cuando la escritura llama a Dios "Señor de los señores" se refiere a que es Señor de realidades que a su vez tienen su señorío sobre lo que es estrictamente sublunar, intramundano, el hombre entre esas realidades. "No es posible por tanto, se hay a querido dar a entender que él sea el señor de todo cuanto se considera como divinidad, de piedra o madera, puesto que no sería glorificar y honrar a Dios, hacerle Señor de la materia... él es el dominador de los dominadores, vale decir de los ángeles, de las esferas celestes".

Incluso la misma formación del mundo es acontecida mediante emanación por estas realidades espirituales que son los ángeles; hace coincidir incluso Maimónides esta visión emanatista con un pasaje de Platón en el cual se dice que Dios mira al mundo de las inteligencias y de él emanó el ser. ${ }^{22}$

22 Ya hemos apuntado como el mundo de las emanaciones entraña —o supone según se mire — un menor contacto de Dios con lo mundano; el mundo va tomando sentido por sí mismo, y, aunque con diferencias aún notables, podemos hablar de un cierto evolucionismo en la mayor parte del emanacionismo árabe y judío. Esta filosofía medieval apuesta por esta cierta autonomía del mundo que tendrá sus especiales consecuencias en el tratamiento del tema de la providencia. 
Tomemos de nuevo la importancia de la profecía en la angelología. Si Maimónides define la profecía como efusión del intelecto activo sobre la facultad racional en primer término y después sobre la imaginativa, es de suponer que el conocimiento de realidades angélicas supone una preparación en estos dos terrenos, tanto en la facultad intelectiva como en la imaginativa, ${ }^{23}$ pero la preparación en la segunda es en orden a plenificar la primera - esto ya se vio en el primer apartado-; saber distinguir entre lo inteligible y lo meramente imaginario es uno de los propósitos primeros de la Guía, así nos lo expone además en uno de los capítulos dedicado a los ángeles; ${ }^{24}$ si el hombre se deja llevar exclusivamente de su imaginación, las falacias cognoscitivas advienen en incontables formas, el conocimiento de los ángeles no está exento de este peligro cognoscitivo. Así que siempre que aparece en la literatura profética la figura angélica se supone que el profeta, precisamente por su categoría de tal está hablando desde esta unión perfecta que hemos mencionado entre la facultad racional y la imaginativa; ahora bien, es gracias a la imaginativa y a una preparación correcta de esta facultad por lo que el revestimiento corporal de los ángeles queda justificado por emplear la Escritura el lenguaje humano, pero tal revestimiento tiene su significado profundo, por ejemplo se hace referencia a la figura de "fuego" en el ángel, este revestimiento corporal indica precisamente el acercamiento a su verdadero ser, de hecho el fuego por naturaleza tiende a ascender, a no tener una figura fija, extensa, medible. ${ }^{25}$ Igualmente acontece cuando el único atributo animal que se le ha insertado al ángel son las alas.

Si seguimos nuestra exposición tal como hasta ahora queda expresada tenemos que al acceder al ángel mediante la visión profética, y no acontecer ésta sino desde una preparación natural del hombre, no podemos decir que es el ángel el que viene al hombre, sino éste el que va al ángel; en una palabra, sólo la profecía posibilitaría visiones angélicas, y aquí nos encontramos con el mismo problema que planteamos al comienzo de este escrito: ¿es el ángel objeto natural de nuestro intelecto?, y la respuesta que forzosamente tenemos que dar ahora es esta: sí, lo es en cuanto es el hombre el que mediante su imaginación y su intelecto accede al ángel. Esta respuesta supone que la profecía es algo natural a todo hombre, pero no sería Dios objeto de nuestro intelecto si consideramos que la profecía, además de implicar esta preparación indicada, necesita de lo que podíamos llamar don de Dios, o lo que igualmente ha venido a llamarse Gracia, y es que en Maimónides está también presente esta segunda visión. Pero la problemática no queda saldada aquí, sino que nos vamos a hacer otra pregunta para responder con más seguridad a la primera, esta segunda pregunta tiene que ver con el para qué de las realidades angélicas, por qué su existencia, ¿fueron creados para dar a conocer algo al hombre?, si esto es así, por qué Dios no cedió el conocimiento directamente al hombre, y así éste con sus facultades podría conocer a Dios, sus designios y sus acciones. Estamos en el problema de los límites que señalamos al comienzo; opino que toda religión opera de esta forma, es decir, en primer lugar la finitud del hombre es puesta de manifiesto con respecto a Dios, su conocimiento es esencialmente diverso al de éste y por tanto necesita de mediaciones para dar 
plenitud a aquello que Kant llamará posteriormente la finalidad intrínseca de la razón humana. Tales mediaciones expresan que son accesibles al hombre una serie de misterios, qué hace entonces el pensador "religioso", muy sencillo, supone el para qué de estas realidades y de esta forma todo lo que es en el universo, lo es de un modo antropocéntrico: ¿qué importancia tienen los ángeles sino la de comunicar al hombre una serie de misterios que a éste le son naturalmente inabarcables?. Ahora tenemos nuestras ganancias y son estas, que el ángel no es algo natural al hombre, ${ }^{26}$ puesto que el ángel existe por algo y ese algo es la comunicabilidad que el ángel tiene que dar al hombre debido a su entendimiento limitado: Dios $\rightarrow$ ángel $\rightarrow$ hombre; visto a la inversa tenemos: el hombre accede al ángel por visión profética, esta necesita del don y el don es dado por Dios, por tanto el ángel no es objeto natural, sino enraizado en un fin que Dios impregna en el hombre: conocerle; Dios tampoco es objeto natural de nuestro entendimiento, puesto que no se da sin el ángel y sin visión profética.

Esto tiene su inmediata comparación con lo que en el hombre es el entendimiento, ¿existe por sí mismo el entendimiento pasivo?, la respuesta de los pensadores medievales es que la existencia del intelecto pasivo tiene su sentido y su plenificación desde el intelecto agente o activo, por tal razón afirma Maimónides que se ha comparado a éste con una realidad angélica. Siguiendo en este parangón, diríamos que el hombre ${ }^{27}$ supone la potencia cognoscitiva y es aquel entendimiento separado, siempre en acto el que hace pasar esta potencia al acto cognoscitivo propiamente dicho.

De ahí que Maimónides considere siempre dos cosas - y además en potencia - el intelecto y el inteligible, ${ }^{28}$ esas dos realidades se unen evidentemente y son la misma cosa (como acontece siempre en el modo de intelección divino) pero mediante el "mediador" que es el intelecto activo.

\section{CONSECUENCIAS METAFÍSICAS DE LA ANGELOLOGÍA EN MAIMÓNIDES. A MODO DE ENSAYO}

Pero volvamos a recoger un pensamiento que habíamos dejado a medio examinar y que en sí engendra la clave de una visión de la angelología esencialmente contraria a la mayor parte de sus antecesores en este particular, donde la figura de Maimónides ejerce una notabilísima influencia posterior en la línea de un judaísmo heterodoxo que llega hasta el mismo Espinosa. ${ }^{29}$ Este pensa-

26 El problema de lo natural en lo que tanto insisto es clave, porque el mismo Maimónides trae constantemente esta lucha entre el ser por naturaleza (en su intento de asimilar a Aristóteles que trató como nadie la "naturaleza del ser") y el ser más allá de la naturaleza; por esto que nadie piense: es que para un creyente la naturaleza humana (del ser) no tiene sentido sino desde la creatio ex nihilo, desde la nihilidad y por tanto naturaleza y gracia se confunden. Este pensamiento del que seguro alguien está tentado, queda vivencialmente superado en Maimónides, que vive una tremenda lucha asimilativa entre puramente la naturaleza del ser y lo "más allá" de la naturaleza.

27 Al referirme al hombre me refiero al alma, que en el caso de Maimónides es coincidente con el intelecto pasivo, no con el activo.

28 Cf. Maimónides: 0.c. I parte, cap.68, p. 182.

29 Nada más allá de mi intención que este escrito dilucide en qué fue o dejó de ser Maimónides un pensador heterodoxo; no obstante e indagando en la angelología, no es la intención de nuestro autor multiplicar sin más la realidad de ángeles; este problema de la excesiva multiplicación de los mismos ya dio origen a fuertes polémicas en la literatura postbíblica e igualmente en la cábala germana con Eleazar de Worms. La multiplicación de los ángeles en Maimónides en ningún momento tiene el carácter que podría entenderse desde lo que son los ángeles: realidades espirituales, mensajeros..., sino que este ver todo lo real como angélico tiene un sentido puramente ontológico y estructuralista, lo veremos de inmediato. 
miento que habíamos dejado no es ni más ni menos que el que nace de esta aseveración ya citada: el ángel como fuerza del movimiento.

Sabido es que el mismo concepto de alma posee en la filosofía griega el doble sentido de ser aquella parte - substancia de hecho- que accedía a las verdades eternas, que se unía con Dios o su derivado; pero además de esto el alma es algo más principal: es principio de vida y de movimiento; y aunque tanto Platón como Aristóteles sostuvieron ambas posturas, el acercarse a esta segunda les daba una mayor consistencia en la línea que los mismos fisiólogos presocráticos habían marcado respecto del eterno movimiento de todos los procesos vivos. Ahora, a finales del siglo XII un autor como Maimónides va a sacar consecuencias tremendas respecto a este punto con su mera afirmación de que los ángeles son fuerzas originarias del movimiento.

Además, este pensamiento no va a venir exento de una polémica jugosa ante la ortodoxia judía. Traigamos este texto para abrirnos camino: "Si dijeras a uno de esos sedicentes sabios de Israel que Dios envía su ángel para que forme dentro de la mujer el feto...lo aceptaría considerándolo una manifestación del poder divino, así como de su sabiduría....

A continuación sigue indagando Maimónides, afirmando además que estas fuerzas realizan una actividad determinada, no dos; esto es puro estructuralismo de lo vivo, en una palabra, es Aristóteles cuando afirma que cada ente persevera en su ser, a nivel de función, de esencia no de existencia, aunque en Aristóteles estos términos no se hallen aún separados- pero aquí el estructuralismo es mayor aún que en Aristóteles, puesto que se habla de "cada fuerza corporal"; esto llega incluso a recordar a sistemas actuales que afirman que más allá de la existencia de substancias, habría sustantividades dentro de lo mal llamado sustancias, donde cada "fuerza corporal es substante en sentido propio". La identificación de los ángeles con las fuerzas vivas posee como digo consecuencias tremendas, y aún hay más.

Afirma nuestro autor que hay ángeles permanentes y perecederos. Para probar este último aserto, más discutible que el primero, ya que "se ha expuesto reiteradamente que son vivientes y estables", trae el siguiente texto perteneciente al Be-resît Rabbâ: "Cada día crea el santo una clase angélica, que entona un cántico ante él y desaparece". ${ }^{30}$ De esta forma pasa nuestro autor a considerar angélica toda realidad, tanto en el orden individual como en el de especies; no encontraremos en toda la Guía a un Maimónides igual a este.

Véase que toda fuerza individual —y el alma es también fuerza-, siendo ella misma angélica, es perecedera. No consta según algunos expertos que todos los escritos de Averroes hayan sido leídos por Maimónides, ${ }^{31}$ pero se asemeja con creces esta postura a la de su conciudadano de infancia sobre el particular. Pero aún no hemos dejado de sorprendernos de afirmaciones como ésta, cuando sigue habiendo más sorpresas y es en su tratamiento de la realidad en cuanto especie. Nos dice que la realidad angélica que es perdurable es la especie, esto nos choca fuertemente cuando

30 Cf. Maimônides: o.c. p.255. ¿qué es la realidad monádica en Leibniz sino esto?. Fuerzas continuamente creadas, aunque véase que el autor del XVII es en esto más idealista que el del XII puesto que esas continuas creaciones son inmortales, no siendo el caso en Maimónides, el estructuralismo en el primero es más fuerte que esta realidad fluyente del autor sefardí; mas el leit motiv es idéntico: preñar la realidad de sentido autónomo, preñarla de fuerzas.

31 Aunque a este respecto nos saca de duda la carta de maimónides a Judá Inb Tibbon, en la que hace referencia a los comentarios de la obra Aristotélica por su conciudadano; cf. La carta citada en : Del Valle, C: Cartas y testamento de Maimónides. Publicaciones del Monte de Piedad y Caja de ahorros de Córdoba; Córdoba, 1989; pp.33-34. 
más adelante podemos leer que tanto las ideas universales como las especies, no tienen realidad fuera del intelecto ${ }^{32} \mathrm{y}$ así tenemos lo siguiente: hay realidades angélicas perecederas de un lado y perpetuas por otro, si lo primero, ya hemos visto que tales realidades serían mortales, si lo segundo, ¿es que el ángel no tiene realidad fuera del intelecto?. Desde esta visión es imposible no ver aquí un materialismo preñado de idealismo radical al modo espinoziano o incluso al modo marxista (curiosamente dos judíos). Es por ello que vamos a centrar ahora nuestra atención en las más que probables influencias de Maimónides en Spinoza respecto a nuestro tema particular.

Es menester respecto a esta intención comparativa entrar en la profundidad de Spinoza. Puesto que el autor judío-portugués no es el tema conciso de este estudio, habré de resumir sus teorías intentando no por ello dejar de tocar el fondo y la inmensa implicación de la metafísica spinoziana.

El mejor concepto que nos ofrece una unión entre estos dos pensadores es el concepto spinoziano de substancia, "la substancia es aquello que existe por sí y no necesita de otro para existir", 33 si tomamos así esta definición podemos decir que nada más ajeno al pensamiento de que cualquier realidad perecedera tiene sentido en y desde sí misma, que es la conclusión que hemos podido vislumbrar en Maimónides. Si además suponemos que esa substancia es identificada por Spinoza con Dios y que todo lo que no sea Dios no tiene realidad substante sino sólo en cuanto que es una parte 0 atributo de Dios, nuestros deseos de ver influencias de la visión adelantada por el autor cordobés en el autor portugués se irían al traste. La riqueza aflora en cambio cuando profundizamos más allá en el mismo concepto spinoziano de substancia. Porque igual se nos está echando abajo el concepto aristotélico de substancia pero hacia unas cotas. ¿Donde está la substancia que es por sí?, pues Spinoza nos dice que ninguna realidad determinada constituye su esencia, así esa substancia que no depende de nada viene a ser absoluta pluralidad e indeterminación. Y acontece algo más respecto de los atributos ya que estos son infinitos en número y todos tienen igual importancia. De esta forma la substancia es una, pero esa unidad significa en lo profundo una infinitud absoluta, tenemos así que la realidad más absoluta, más en sí, no es nada determinado; y hay quien a esto lo llama panteísmo y no un puro naturalismo de corte pagano; resonancias a esto mismo también las encontramos en $\mathrm{N}$. Hartmann cuando afirma que lo necesario no es padre de lo contingente sino al revés, en Anaximandro cuando dice que lo determinado es hijo de lo indeterminado. Además este indeterminismo viene a tocar la raíz de otra idea maimonídea y es aquella que afirma que"el ángel nunca da la esencia de Dios, sino tan sólo acciones de él". Así tenemos que no se conoce la esencia de Dios sino sólo sus acciones. ${ }^{34}$ Tenemos un Dios menos estático, más activo, cobrando toda actividad mediante el ángel - un carácter de divinidad, pero a la vez, en su esencia es Dios más incognoscible. Que además nuestro autor hable de fuerzas activas en una cosa, imperceptibles al sentido, que cada fuerza corporal sea un ángel y que tales están difundidos por todo el universo suena a la expansión de los infinitos atributos numéricos de la realidad en Spinoza, ${ }^{35}$ lo realmente importante en éste es la infinita solidez de los atributos y no de la substancia. La substancia es tan sólo el argumento

32 Cf. Maimónides:o.c. III parte, cap.18, p.417.

33 Cf. Spinoza: Ética demostrada según el orden geométrico. Ed. Nacional, Madrid, 1980, p.47.

34 Este tema del conocimiento de Dios por sus acciones y no por sus atributos ni por su esencia (al hombre le está vedado ver a Dios "cara a cara") puede verse especialmente en los cap 53, 59-61 de la I parte de la Guía de perplejos.

35 Spinoza: 0.c. p.112-114. 
lógico que halla unidad en la multilicidad, como el logos de Heráclito da sentido y orden al cambio continuo, pero tanto éste como los infinitos y substantes atributos son la realidad real. Spinoza ha hecho del concepto de substancia el más contingente de todos, rompiendo así con la primacía de la necesidad y estatismo que tal concepto poseyó siempre en la filosofía. Y maimónides ha hecho del concepto de Dios el más indeterminado en su esencia, lo realmente determinado, portador de sentido diría un contemporáneo son ni más ni menos que estas realidades angélicas que se encuentran en cada estrato de la realidad. Pues bien, este naturalismo ¿no es el mismo que defiende Maimónides en estos capítulos dedicados a la realidad angélica?.

Antonio Ríos Rojas 